\title{
Introduction to Stopping Time in Stochastic Finance Theory. Part II
}

\author{
Peter Jaeger \\ Siegmund-Schacky-Str. 18a \\ 80993 Munich, Germany
}

Summary. We start proceeding with the stopping time theory in discrete time with the help of the Mizar system 1, 4. We prove, that the expression for two stopping times $k_{1}$ and $k_{2}$ not always implies a stopping time $\left(k_{1}+k_{2}\right)$ (see Theorem 6 in this paper). If you want to get a stopping time, you have to cut the function e.g. $\left(k_{1}+k_{2}\right) \cap T$ (see [2, p. 283 Remark 6.14]).

Next we introduce the stopping time in continuous time. We are focused on the intervals $[0, r]$ where $r \in \mathbb{R}$. We prove, that for $I=[0, r]$ or $I=[0,+\infty[$ the set $\{A \cap I: A \in$ Borel-Sets $\}$ is a $\sigma$-algebra of $I$ (see Definition 6 in this paper, and more general given in [3, p.12 1.8e]). The interval $I$ can be considered as a timeline from now to some point in the future.

This set is necessary to define our next lemma. We prove the existence of the $\sigma$-algebra of the $\tau$-past, where $\tau$ is a stopping time (see Definition 11 in this paper and [6, p.187, Definition 9.19]). If $\tau_{1}$ and $\tau_{2}$ are stopping times with $\tau_{1}$ is smaller or equal than $\tau_{2}$ we can prove, that the $\sigma$-algebra of the $\tau_{1}$-past is a subset of the $\sigma$-algebra of the $\tau_{2}$-past (see Theorem 9 in this paper and [6, p.187 Lemma 9.21]).

Suppose, that you want to use Lemma 9.21 with some events, that never occur, see as a comparison the paper [5] and the example for $\operatorname{ST}(1)=\{+\infty\}$ in the Summary. We don't have the element $+\infty$ in our above-mentioned time intervals $[0, r[$ and $[0,+\infty[$. This is only possible if we construct a new $\sigma$-algebra on $\mathbb{R} \cup\{-\infty,+\infty\}$. This construction is similar to the Borel-Sets and we call this $\sigma$-algebra extended Borel sets (see Definition 13 in this paper and 3 p. 21]). It can be proved, that $\{+\infty\}$ is an Element of extended Borel sets (see Theorem 21 in this paper). Now we use the interval $[0,+\infty]$ as a basis. We construct a $\sigma$-algebra on $[0,+\infty]$ similar to the book ([3, p. $1218 \mathrm{e}]$ ), see Definition 18 in this paper, and call it extended Borel subsets. We prove for stopping times with this given $\sigma$-algebra, that for $\tau_{1}$ and $\tau_{2}$ are stopping times with $\tau_{1}$ is smaller or equal than $\tau_{2}$ we have the $\sigma$-algebra of the $\tau_{1}$-past is a subset of the $\sigma$-algebra of the $\tau_{2}$-past, see Theorem 25 in this paper. It is obvious, that $\{+\infty\} \in$ extended Borel subsets. 
In general, Lemma 9.21 is important for the proof of the Optional Sampling Theorem, see 10.11 Proof of (i) in [6] p. 203].

MSC: 60G40 03B35

Keywords: stopping time; stochastic process

MML identifier: FINANCE5, version: 8.1.06 5.45.1311

\section{Preliminaries}

From now on $\Omega$ denotes a non empty set, $\Sigma$ denotes a $\sigma$-field of subsets of $\Omega, S$ denotes a non empty subset of $\mathbb{R}, r$ denotes a real number, and $T$ denotes a natural number.

Let $A$ be a non empty set, $I$ be an extended real-membered set, and $k_{1}, k_{2}$ be functions from $A$ into $I$. We say that $k_{1} \leqslant k_{2}$ if and only if

(Def. 1) for every element $w$ of $A, k_{1}(w) \leqslant k_{2}(w)$.

Let $f_{1}, f_{2}$ be extended real-valued functions. The functor $f_{1}+f_{2}$ yielding a function is defined by

(Def. 2) $\operatorname{dom} i t=\operatorname{dom} f_{1} \cap \operatorname{dom} f_{2}$ and for every object $x$ such that $x \in \operatorname{dom} i t$ holds it $(x)=f_{1}(x)+f_{2}(x)$.

One can check that the functor is commutative.

Let us note that $f_{1}+f_{2}$ is extended real-valued.

Let $C$ be a set, $D_{1}, D_{2}$ be extended real-membered, non empty sets, $f_{1}$ be a function from $C$ into $D_{1}$, and $f_{2}$ be a function from $C$ into $D_{2}$. One can verify that $f_{1}+f_{2}$ is total as a partial function from $C$ to $\overline{\mathbb{R}}$.

Let $D_{1}, D_{2}$ be extended real-membered sets, $f_{1}$ be a partial function from $C$ to $D_{1}$, and $f_{2}$ be a partial function from $C$ to $D_{2}$. Let us note that the functor $f_{1}+f_{2}$ yields a partial function from $C$ to $\overline{\mathbb{R}}$. Now we state the propositions:

(1) Let us consider non empty sets $A, I, y$, and a function $F$ from $A$ into $I$. Then $\{z$, where $z$ is an element of $A: F(z) \in y\}=F^{-1}(y)$.

(2) Let us consider a real number $r$. If $r>0$, then there exists a natural number $n$ such that $\frac{1}{n}<r$ and $n>0$.

(3) Let us consider real numbers $a, b$. Then $[-\infty, a] \cap[b,+\infty]=[b, a]$.

(4) Let us consider a real number $r$. Suppose $r \geqslant 0$. Then $[0,+\infty] \backslash[0, r[=$ $[r,+\infty]$.

Let $r$ be an extended real. Observe that $[r,+\infty]$ is non empty.

(5) Let us consider an extended real $k$. Then $\overline{\mathbb{R}} \backslash[-\infty, k]=] k,+\infty]$.

Let $a$ be a real number. One can check that $] a,+\infty]$ is non empty. 


\section{Stopping Time in Discrete Time}

Let us consider $\Omega, \Sigma$, and $T$. Let $F_{1}$ be a filtration of $\bigcup_{t \in \mathbb{N}: 0 \leqslant t \leqslant T}\{t\}$ and $\Sigma$ and $k$ be a function from $\Omega$ into $T_{\{+\infty\}}$. We say that $k$ is like stopping time of $F_{1}$ if and only if

(Def. 3) $k$ is $\operatorname{StoppingTime}\left(F_{1}, T\right)$.

Let $M_{1}$ be a filtration of $\bigcup_{t \in \mathbb{N}: 0 \leqslant t \leqslant T}\{t\}$ and $\Sigma$. Note that there exists a function from $\Omega$ into $T_{\{+\infty\}}$ which is like stopping time of $M_{1}$.

A stopping time of $M_{1}$ is a like stopping time of $M_{1}$ function from $\Omega$ into $T_{\{+\infty\}}$. Now we state the proposition:

(6) Let us consider a non zero natural number $T$, and a filtration $M_{1}$ of $\bigcup_{t \in \mathbb{N}: 0 \leqslant t \leqslant T}\{t\}$ and $\Sigma$. Then there exist stopping times $k_{1}, k_{2}$ of $M_{1}$ such that $k_{1}+k_{2}$ is not a stopping time of $M_{1}$.

Proof: Reconsider $M_{2}=T$ as an element of $T_{\{+\infty\}}$. Consider $k_{1}$ being a function from $\Omega$ into $T_{\{+\infty\}}$ such that $k_{1}=\Omega \longmapsto M_{2}$ and $k_{1}$ is Stopping Time $\left(M_{1}, T\right)$. Consider $k_{2}$ being a function from $\Omega$ into $T_{\{+\infty\}}$ such that $k_{2}=\Omega \longmapsto M_{2}$ and $k_{2}$ is $\operatorname{StoppingTime}\left(M_{1}, T\right)$. There exists an element $w$ of $\operatorname{dom}\left(k_{1}+k_{2}\right)$ such that $w \in \operatorname{dom}\left(k_{1}+k_{2}\right)$ and $\left(k_{1}+k_{2}\right)(w) \notin T_{\{+\infty\}}$.

\section{Stopping Time in Continuous Time}

Let $r$ be a real number.

A stopping event of $r$ is a subset of $\mathbb{R}$ defined by

(Def. 4)

(i) it $=[0,+\infty[$, if $r \leqslant 0$,

(ii) it $=[0, r]$, otherwise.

Let us note that every stopping event of $r$ is non empty.

In the sequel $I$ denotes a stopping event of $r$.

Now we state the proposition:

(7) $I$ is an event of the Borel sets.

\section{Borel-SETS}

Let us consider $r$ and $I$. Let $A$ be an element of the Borel sets. The intersection of $A$ and $I$ yielding an element of the Borel sets is defined by (Def. 5) $A \cap I$.

The first Borel subsets with $I$ yielding a $\sigma$-field of subsets of $I$ is defined by 
(Def. 6) the set of all the intersection of $A$ and $I$ where $A$ is an element of the Borel sets.

Let us consider $\Omega$ and $\Sigma$. Let $M_{1}$ be a function and $k$ be a random variable of $\Sigma$ and the first Borel subsets with $I$. We say that $k$ is stopping time of $M_{1}$ if and only if

(Def. 7) for every element $t$ of $I,\{w$, where $w$ is an element of $\Omega: k(w) \leqslant t\} \in$ $M_{1}(t)$.

(8) Let us consider a filtration $M_{1}$ of $I$ and $\Sigma$, and an element $t_{1}$ of $I$. Then there exists a random variable $q$ of $\Sigma$ and the first Borel subsets with $I$ such that

(i) $q=\Omega \longmapsto t_{1}$, and

(ii) $q$ is stopping time of $M_{1}$.

Proof: For every element $t$ of $I,\{w$, where $w$ is an element of $\Omega:(\Omega \longmapsto$ $\left.\left.t_{1}\right)(w) \leqslant t\right\} \in M_{1}(t)$. Set $O=\Omega \longmapsto t_{1}$. For every set $x, O^{-1}(x) \in \Sigma$.

Let us consider $\Omega, \Sigma, r$, and $I$. Let $F_{1}$ be a filtration of $I$ and $\Sigma$ and $k$ be a random variable of $\Sigma$ and the first Borel subsets with $I$. We say that $k$ is like stopping time of $F_{1}$ if and only if

(Def. 8) $\quad k$ is stopping time of $F_{1}$.

Let $M_{1}$ be a filtration of $I$ and $\Sigma$. One can check that there exists a random variable of $\Sigma$ and the first Borel subsets with $I$ which is like stopping time of $M_{1}$.

A stopping time of $M_{1}$ is a like stopping time of $M_{1}$ random variable of $\Sigma$ and the first Borel subsets with $I$.

\section{5. $\sigma$-AlgeBRA OF THE $\tau$-PAST}

Let us consider $\Omega, \Sigma, r$, and $I$. Let $M_{1}$ be a filtration of $I$ and $\Sigma, \tau$ be a stopping time of $M_{1}$, and $A_{1}$ be a sequence of subsets of $\Omega$. Assume $\operatorname{rng} A_{1} \subseteq$ $\left\{A\right.$, where $A$ is an element of $\Sigma$ : for every element $t_{1}$ of $I, A \cap\{w$, where $w$ is an element of $\left.\left.\Omega: \tau(w) \leqslant t_{1}\right\} \in M_{1}\left(t_{1}\right)\right\}$. Let $t$ be an element of $I$ and $n$ be a natural number. The first set for $\sigma$-tau of $\tau, A_{1}, n$ and $t$ yielding an element of the $t-\mathcal{E} \mathcal{F}$ of $M_{1}$ is defined by the term

(Def. 9) (Complement $\left.A_{1}\right)(n) \cap\{w$, where $w$ is an element of $\Omega: \tau(w) \leqslant t\}$.

Let $A$ be a sequence of subsets of $\Omega$. The second set for $\sigma$-tau of $\tau, A$ and $t$ yielding a sequence of subsets of the $t-\mathcal{E F}$ of $M_{1}$ is defined by

(Def. 10) for every natural number $n$, it $(n)=$ the first set for $\sigma$-tau of $\tau, A, n$ and $t$. 
The functor $\Sigma-\operatorname{tau}(\tau)$ yielding a $\sigma$-field of subsets of $\Omega$ is defined by the term

(Def. 11) $\{A$, where $A$ is an element of $\Sigma$ : for every element $t$ of $I, A \cap\{w$, where $w$ is an element of $\left.\Omega: \tau(w) \leqslant t\} \in M_{1}(t)\right\}$.

Now we state the proposition:

(9) Let us consider a filtration $M_{1}$ of $I$ and $\Sigma$, and stopping times $k_{1}, k_{2}$ of $M_{1}$. Suppose $k_{1} \leqslant k_{2}$. Then $\Sigma-\operatorname{tau}\left(k_{1}\right) \subseteq \Sigma$-tau $\left(k_{2}\right)$.

Proof: Consider $A$ being an element of $\Sigma$ such that $x=A$ and for every element $t$ of $I, A \cap\left\{w_{1}\right.$, where $w_{1}$ is an element of $\Omega: k_{1}\left(w_{1}\right) \leqslant$ $t\} \in M_{1}(t) . x \in\{A$, where $A$ is an element of $\Sigma$ : for every element $t$ of $I, A \cap\left\{w_{1}\right.$, where $w_{1}$ is an element of $\left.\left.\Omega: k_{2}\left(w_{1}\right) \leqslant t\right\} \in M_{1}(t)\right\}$.

The extended family of halflines yielding a family of subsets of $\overline{\mathbb{R}}$ is defined by the term

(Def. 12) the set of all $[-\infty, r]$ where $r$ is a real number.

The extended Borel sets yielding a $\sigma$-field of subsets of $\overline{\mathbb{R}}$ is defined by the term

(Def. 13) $\sigma$ (the extended family of halflines).

Now we state the proposition:

(10) Let us consider a real number $k$. Then

(i) $] k,+\infty]$ is an element of the extended Borel sets, and

(ii) $[-\infty, k]$ is an element of the extended Borel sets.

The theorem is a consequence of (5).

Let $b$ be a real number. The extended half open sets of $b$ yielding a sequence of subsets of $\overline{\mathbb{R}}$ is defined by

(Def. 14) $i t(0)=] b-1,+\infty]$ and for every natural number $n$, it $(n+1)=] b-$ $\left.\frac{1}{n+1},+\infty\right]$.

Let us consider a real number $b$. Now we state the propositions:

(11) Intersection(the extended half open sets of $b$ ) is an element of the extended Borel sets.

PROOF: For every natural number $n$, (Complement(the extended half open sets of $b)(n)$ is an element of the extended Borel sets.

(12) Intersection(the extended half open sets of $b)=[b,+\infty]$.

Proof: For every object $c, c \in$ Intersection(the extended half open sets of $b)$ iff $c \in[b,+\infty]$.

(13) Let us consider real numbers $a, b$. Then $[b, a]$ is an element of the extended Borel sets.

Proof: $[-\infty, a]$ is an element of the extended Borel sets. $[-\infty, a] \cap[b,+\infty]$ is an element of the extended Borel sets by (12), (11), [7, (19)]. 
(14) Let us consider a real number $a$. Then $\{a\}$ is an element of the extended Borel sets. The theorem is a consequence of (13).

(15) Let us consider a real number $r$. Then $[r,+\infty]$ is an event of the extended Borel sets. The theorem is a consequence of (11) and (12).

Let $b$ be a real number. The extended right closed sets of $b$ yielding a sequence of subsets of $\overline{\mathbb{R}}$ is defined by

(Def. 15) for every natural number $n$, it $(n)=[-\infty, b-n]$.

Now we state the propositions:

(16) Let us consider a real number $b$. Then Intersection(the extended right closed sets of $b$ ) is an element of the extended Borel sets. The theorem is a consequence of (10).

(17) Intersection(the extended right closed sets of 0$)=\{-\infty\}$.

Proof: For every object $c, c \in$ Intersection(the extended right closed sets of 0$)$ iff $c \in\{-\infty\}$.

(18) $\{-\infty\}$ is an element of the extended Borel sets.

Let $b$ be a real number. The extended left closed sets of $b$ yielding a sequence of subsets of $\overline{\mathbb{R}}$ is defined by

(Def. 16) for every natural number $n$, it $(n)=[b+n,+\infty]$.

Now we state the propositions:

(19) Let us consider a real number $b$. Then Intersection(the extended left closed sets of $b$ ) is an element of the extended Borel sets. The theorem is a consequence of (15).

(20) Intersection(the extended left closed sets of 0$)=\{+\infty\}$.

Proof: For every object $c, c \in$ Intersection(the extended left closed sets of 0$)$ iff $c \in\{+\infty\}$.

(21) $\{+\infty\}$ is an element of the extended Borel sets.

(22) $\mathbb{R}$ is an element of the extended Borel sets. The theorem is a consequence of (19), (20), (16), (17), and (2).

(23) Halflines $\subseteq$ the extended Borel sets. The theorem is a consequence of (10), (14), (16), and (17).

Let $A$ be an element of the extended Borel sets. The positive subset of $A$ yielding an element of the extended Borel sets is defined by the term

(Def. 17) $\quad A \cap[0,+\infty]$.

The extended Borel subsets yielding a $\sigma$-field of subsets of $[0,+\infty]$ is defined by the term

(Def. 18) the set of all the positive subset of $A$ where $A$ is an element of the extended Borel sets. 
Now we state the proposition:

(24) $\{+\infty\}$ is an element of the extended Borel subsets. The theorem is a consequence of $(21)$.

Let us consider $\Omega$ and $\Sigma$. Let $M_{1}$ be a function, $S$ be a non empty, extended real-membered set, and $k$ be a random variable of $\Sigma$ and the extended Borel subsets. We say that $k$ is $\operatorname{StoppingTime}\left(M_{1}, S\right)$ if and only if

(Def. 19) for every element $t$ of $S,\{w$, where $w$ is an element of $\Omega: k(w) \leqslant t\} \in$ $M_{1}(t)$.

Now we state the proposition:

(25) Let us consider a filtration $M_{1}$ of $S$ and $\Sigma$, and an element $t_{1}$ of $[0,+\infty]$. Then there exists a random variable $q$ of $\Sigma$ and the extended Borel subsets such that

(i) $q=\Omega \longmapsto t_{1}$, and

(ii) $q$ is Stopping Time $\left(M_{1}, S\right)$.

Proof: For every element $t$ of $S,\{w$, where $w$ is an element of $\Omega:(\Omega \longmapsto$ $\left.\left.t_{1}\right)(w) \leqslant t\right\} \in M_{1}(t)$. Set $O=\Omega \longmapsto t_{1}$. For every set $x, O^{-1}(x) \in \Sigma$.

Let us consider $\Omega, \Sigma$, and $S$. Let $F_{1}$ be a filtration of $S$ and $\Sigma$ and $k$ be a random variable of $\Sigma$ and the extended Borel subsets. We say that $k$ is like stopping time of $F_{1}$ if and only if

(Def. 20) $k$ is $\operatorname{StoppingTime}\left(F_{1}, S\right)$.

Let $M_{1}$ be a filtration of $S$ and $\Sigma$. Observe that there exists a random variable of $\Sigma$ and the extended Borel subsets which is like stopping time of $M_{1}$.

A stopping time of $\Sigma$ and $M_{1}$ is a like stopping time of $M_{1}$ random variable of $\Sigma$ and the extended Borel subsets. Let $\tau$ be a stopping time of $\Sigma$ and $M_{1}$ and $A_{1}$ be a sequence of subsets of $\Omega$. Assume $\operatorname{rng} A_{1} \subseteq\{A$, where $A$ is an element of $\Sigma$ : for every element $t_{1}$ of $S, A \cap\{w$, where $w$ is an element of $\Omega: \tau(w) \leqslant$ $\left.\left.t_{1}\right\} \in M_{1}\left(t_{1}\right)\right\}$. Let $t$ be an element of $S$ and $n$ be a natural number. The first set for $\sigma$-tau of $M_{1}, \tau, A_{1}, n$ and $t$ yielding an element of the $t$ - $\mathcal{E} \mathcal{F}$ of $M_{1}$ is defined by the term

(Def. 21) (Complement $\left.A_{1}\right)(n) \cap\{w$, where $w$ is an element of $\Omega: \tau(w) \leqslant t\}$.

The second set for $\sigma$-tau of $M_{1}, \tau, A_{1}$ and $t$ yielding a sequence of subsets of the $t-\mathcal{E} \mathcal{F}$ of $M_{1}$ is defined by

(Def. 22) for every natural number $n$, it $(n)=$ the first set for $\sigma$-tau of $M_{1}, \tau, A_{1}$, $n$ and $t$.

The functor $\Sigma-\operatorname{tau}\left(M_{1}, \tau\right)$ yielding a $\sigma$-field of subsets of $\Omega$ is defined by the term

(Def. 23) $\{A$, where $A$ is an element of $\Sigma$ : for every element $t$ of $S, A \cap\{w$, where $w$ is an element of $\left.\Omega: \tau(w) \leqslant t\} \in M_{1}(t)\right\}$. 
Now we state the proposition:

(26) Let us consider a filtration $M_{1}$ of $S$ and $\Sigma$, and stopping times $k_{1}, k_{2}$ of $\Sigma$ and $M_{1}$. Suppose $k_{1} \leqslant k_{2}$. Then $\Sigma-\operatorname{tau}\left(M_{1}, k_{1}\right) \subseteq \Sigma-\operatorname{tau}\left(M_{1}, k_{2}\right)$.

Proof: Consider $A$ being an element of $\Sigma$ such that $x=A$ and for every element $t$ of $S, A \cap\left\{w_{1}\right.$, where $w_{1}$ is an element of $\Omega: k_{1}\left(w_{1}\right) \leqslant$ $t\} \in M_{1}(t)$. For every element $t$ of $S, x \cap\left\{w_{1}\right.$, where $w_{1}$ is an element of $\left.\Omega: k_{2}\left(w_{1}\right) \leqslant t\right\} \in M_{1}(t)$.

\section{REFERENCES}

[1] Grzegorz Bancerek, Czesław Byliński, Adam Grabowski, Artur Korniłowicz, Roman Matuszewski, Adam Naumowicz, Karol Pąk, and Josef Urban. Mizar: State-of-the-art and beyond. In Manfred Kerber, Jacques Carette, Cezary Kaliszyk, Florian Rabe, and Volker Sorge, editors, Intelligent Computer Mathematics, volume 9150 of Lecture Notes in Computer Science, pages 261-279. Springer International Publishing, 2015. ISBN 978-3319-20614-1. doi 10.1007/978-3-319-20615-8_17.

[2] Hans Föllmer and Alexander Schied. Stochastic Finance: An Introduction in Discrete Time, volume 27 of Studies in Mathematics. de Gruyter, Berlin, 2nd edition, 2004.

[3] Hans-Otto Georgii. Stochastik, Einführung in die Wahrscheinlichkeitstheorie und Statistik. deGruyter, Berlin, 2nd edition, 2004.

[4] Adam Grabowski, Artur Korniłowicz, and Adam Naumowicz. Four decades of Mizar. Journal of Automated Reasoning, 55(3):191-198, 2015. doi:10.1007/s10817-015-9345-1

[5] Peter Jaeger. Introduction to stopping time in stochastic finance theory. Formalized Mathematics, 25(2):101-105, 2017. doi 10.1515/forma-2017-0010

[6] Achim Klenke. Wahrscheinlichkeitstheorie. Springer-Verlag, Berlin, Heidelberg, 2006.

[7] Andrzej Nędzusiak. $\sigma$-fields and probability. Formalized Mathematics, 1(2):401-407, 1990.

Received November 29, 2017

The English version of this volume of Formalized Mathematics was financed under agreement 548/P-DUN/2016 with the funds from the Polish Minister of Science and Higher Education for the dissemination of science. 